BMJ Open Diabetes Research $\&$ Care

\section{Post hoc efficacy and safety analysis of insulin glargine/lixisenatide fixed- ratio combination in North American patients compared with the rest of world}

To cite: Dailey G, Bajaj HS, Dex T, et al. Post hoc efficacy and safety analysis of insulin glargine/lixisenatide fixedratio combination in North American patients compared with the rest of world. BMJ Open Diab Res Care 2019;7:e000581. doi:10.1136/ bmjdrc-2018-000581

Received 18 July 2018 Revised 7 December 2018 Accepted 9 December 2018

Check for updates

C Author(s) (or their employer(s)) 2019. Re-use permitted under CC BY-NC. No commercial re-use. See rights and permissions. Published by BMJ.

${ }^{1}$ Division of Diabetes and Endocrinology, Scripps Whittier Diabetes Institute, San Diego, California, USA

${ }^{2}$ LMC Diabetes and Endocrinology, Brampton, Ontario, Canada

${ }^{3}$ Leadership Sinai Centre for Diabetes, Mount Sinai Hospital, Toronto, Ontario, Canada ${ }^{4}$ Sanofi US, Bridgewater, New Jersey, USA

${ }^{5}$ Sanofi Canada, Laval, Quebec, Canada

${ }^{6}$ Eastern Virginia Medical School, Norfolk, Virginia, USA

Correspondence to Dr George Dailey; dailey.george@scrippshealth. org

\section{ABSTRACT}

Objective To assess the efficacy and safety of iGlarLixi (titratable fixed-ratio combination of insulin glargine (iGlar) and lixisenatide) in patients with type 2 diabetes (T2D) living in North America (NA; USA and Canada) compared with the rest of the world (RoW).

Research design and methods Post hoc analysis included patient-level data from 509 sites/centers across two phase III trials: LixiLan-0 (NCT02058147; insulin-naive patients; NA, $n=371$; RoW, $n=796$ ) and LixiLan-L (NCT02058160; inadequately controlled patients on basal insulin; NA, $n=196$; RoW, $n=535$ ). Efficacy outcomes were: change from baseline to Week 30 in glycated hemoglobin (HbA1c), postprandial glucose (PPG), PPG excursions, fasting plasma glucose (FPG) and body weight; proportion of patients achieving $\mathrm{HbA} 1 \mathrm{c}<42 \mathrm{mmol} / \mathrm{mol}(<7.0 \%)$; proportion of patients achieving composite endpoint: $\mathrm{HbA} 1 \mathrm{c}<42$ $\mathrm{mmol} / \mathrm{mol}(<7.0 \%)$, no weight gain or symptomatic hypoglycemia (blood glucose $\leq 3.9 \mathrm{mmol} / \mathrm{L}$ ( $\leq 70$ $\mathrm{mg} / \mathrm{dL})$ ). Safety endpoints included incidence of documented symptomatic hypoglycemia and gastrointestinal (Gl) adverse events.

Results Significantly larger reductions $(p \leq 0.003)$ in $\mathrm{HbA1c}$ from baseline to Week 30 were achieved with iGlarLixi, compared with iGlar or lixisenatide, in NA and RoW patients in LixiLan-0 (iGlarLixi vs iGlar: -0.31 and -0.29 , respectively; iGlarLixi vs lixisenatide: -0.84 and -0.69 , respectively) and in LixiLan-L (iGlarLixi vs iGlar: -0.5 and -0.51 , respectively). Documented symptomatic hypoglycemia was similar between NA and RoW patients. iGlarLixi resulted in significant weight benefits versus iGlar (change from baseline -1.58 and $-1.29 \mathrm{~kg}$ for NA and RoW patients, respectively; $\mathrm{p}<0.001$ ). Gl adverse events were similar for iGlarLixi and iGlar, but significantly higher for lixisenatide. Conclusions iGlarLixi improved glycemic parameters versus iGlar or lixisenatide alone in both NA and RoW patients, with beneficial weight effects versus iGlar. iGlarLixi treatment responses, hypoglycemia risk and $\mathrm{Gl}$ adverse events in NA patients were comparable with patients in the RoW.

Trial registry Clinicaltrials.gov NCT02058147 and NCT02058160.-

\section{Significance of the study}

What is already known about this subject?

- iGlarLixi has demonstrated greater efficacy (HbA1c reduction) and safety (no additional risk of hypoglycemia) in phase II and III clinical trials compared with iGlar alone and fewer gastrointestinal adverse events compared with lixisenatide.

What are the new findings?

- This post hoc study compared treatment outcomes for subjects in the North America region with those from the rest of the world utilizing patient-level data from 509 sites/centers across two phase III trials.

How might these results change the focus of research or clinical practice?

- Despite differences in baseline characteristics, outcomes were broadly similar in the two geographic groups, indicating the applicability of clinical trial results of iGlarLixi to North American patients.

\section{INTRODUCTION}

Type 2 diabetes (T2D) represents a global health challenge, with an alarming increase in prevalence worldwide. Development of T2D can be impacted by a range of factors, including diet, lifestyle and genetic predisposition. These factors vary in different races and ethnic groups, ${ }^{1}$ and regional variations can also impact the response to treatment and outcomes in T2D. ${ }^{2-4}$ For example, response to insulin therapy appears to be particularly poor in Asian Indians versus non-Asians, ${ }^{2}$ while African Americans and Hispanics show poorer glycemic control and experience a higher rate of diabetes-related complications versus non-Hispanic whites. ${ }^{3}$ Key factors known to impact on outcome and treatment vary between geographic areas, including obesity, cultural norms regarding 
diet, lifestyle and other health behaviors, prevailing treatment guidelines for glycemic control, blood pressure, lipids and cardiovascular management, as well as funding and nature of healthcare systems. Baseline differences, such as duration of diabetes, body weight and HbAlc, between US participants and Europeans have been reported in a study investigating risk factors for cardiovascular mortality and morbidity. ${ }^{5}$ The authors concluded that variation in regional cardiovascular risk factor management targets may result in these differences, and may therefore also contribute to observed differences in drug efficacy between regions.

Early optimal glycemic control and maintenance has been shown to prevent the development and progression of diabetes-related microvascular complications, and is a key treatment goal. ${ }^{6-8}$ As a progressive disease, T2D requires constant monitoring and timely treatment intensification, necessitating the introduction of glucagon-like peptide 1 receptor agonists (GLP-1 RA) and/or basal and/or bolus insulin following initial therapy with oral antidiabetic drugs (OADs) ${ }^{6-9}$ Real-world evidence suggests that if patients on basal insulin do not achieve glycemic control at 12 months after basal insulin initiation, they are unlikely to do so thereafter (IBM Watson). The ominous octet defines the multi-organ contribution to hyperglycemia, giving rise to the need for multiple pharmacologic options that target specific physiologic defects. ${ }^{10}$ Combination therapies known to have complementary mechanisms of action represent one approach to correcting specific glycemic disturbances. Recent studies have demonstrated that adding GLP-1 RA to basal insulin, either individually or as a fixed-ratio combination, results in equally effective or slightly superior glycemic control, with additional benefits of reduced to similar hypoglycemia, fewer GI adverse events compared with GLP-1 RA use alone, and weight loss. ${ }^{11-13}$

Insulin glargine/lixisenatide (iGlarLixi) is a once-daily titratable, fixed-ratio combination of insulin glargine 100 units/mL (iGlar) and the GLP-1 RA lixisenatide approved by the Food and Drug Administration and the European Medicines Agency. In the European Union, iGlarLixi is indicated in combination with metformin for the treatment of adults with T2D to improve glycemic control when this has not been provided by metformin alone or metformin combined with another OAD or with basal insulin. In the USA, iGlarLixi is indicated in patients unable to achieve glycemic control while on basal insulin $<60 \mathrm{U}$ or lixisenatide. The rationale for the development of iGlarLixi was to combine the complementary actions of iGlar, which primarily improves fasting plasma glucose (FPG) levels, ${ }^{14}$ and lixisenatide, which affects postprandial glucose (PPG) levels by a glucose-dependent stimulation of insulin and suppression of glucagon. In addition, lixisenatide slows gastric emptying, thereby lessening PPG excursions. ${ }^{15-17}$ iGlarLixi has demonstrated efficacy and safety in phase II and III clinical trials. ${ }^{18-20}$ In two large multinational phase III clinical trials, once-daily iGlarLixi demonstrated greater reductions in $\mathrm{HbAlc}$ with no additional risk of hypoglycemia versus iGlar alone in insulin-naive patients inadequately controlled on OADs (LixiLan-O) ${ }^{16}$ and versus iGlar alone in insulin-experienced patients inadequately controlled on basal insulin \pm metformin (LixiLan-L). ${ }^{18}$

The USA and Canada are reported as having the highest and third highest global age-standardized diabetes prevalence, respectively, with disease-related healthcare expenditure higher than the rest of the world (RoW) combined. ${ }^{21}$ Given this high disease and economic burden, and the impact of ethnic and cultural differences on T2D risk, the analysis of treatment options within the North American population can provide useful insight for individualization of therapy within this region. In this post hoc analysis using patient-level data from the LixiLan-O and LixiLan-L randomized controlled trials (RCTs), we compared efficacy and safety outcomes, as well as treatment regimens, for patients treated with iGlarLixi, iGlar and lixisenatide in North America (NA; USA and Canada) compared with patients in the RoW.

\section{PATIENTS AND METHODS}

\section{Study design}

This was a post hoc analysis of patient-level data from two 30-week treatment, phase III clinical trials: LixiLan-O $(\text { NCT02058147 })^{20}$ and LixiLan-L (NCT02058160). ${ }^{18}$

LixiLan-O investigated the efficacy and safety of iGlarLixi versus iGlar and lixisenatide alone in patients with T2D inadequately controlled on metformin monotherapy or metformin combined with a second $\mathrm{OAD}$, which was discontinued at study entry. The study design has previously been described elsewhere. ${ }^{20}$

LixiLan-L investigated the efficacy and safety of iGlarLixi versus iGlar in patients with T2D inadequately controlled on basal insulin without or with $\leq 2$ OADs; all patients entered a 6 -week run-in phase during which any $\mathrm{OAD}$ other than metformin was stopped, patients were switched to iGlar (if they had previously been receiving another basal insulin), and the daily dose of iGlar was titrated and/or stabilized for all patients. Eligible patients were then randomized to either iGlar or iGlarlixi. The study design has previously been described elsewhere. ${ }^{18}$

For the purpose of this analysis, patients were divided into two groups for each study: NA for those patients treated in trial centers in the USA and Canada and RoW for patients from all other countries (Australia, Belgium, Chile, Czech Republic, Denmark, Estonia, France, Germany, Hungary, Italy, Latvia, Lithuania, Mexico, Netherlands, Poland, Romania, Russian Federation, Slovakia, South Africa, Spain, Sweden, Ukraine, UK).

\section{Study endpoints}

The main efficacy outcome measures for the analysis were: change from baseline to Week 30 in HbA1c, PPG (measured 2 hours after standard liquid meal in all patients administering the investigational agents in the morning), PPG excursions, FPG, and body weight; proportion of patients 
achieving HbAlc $<42 \mathrm{mmol} / \mathrm{mol}(<7.0 \%)$; proportion of patients achieving composite endpoint of HbAlc $<42$ $\mathrm{mmol} / \mathrm{mol}(<7.0 \%)$, no weight gain or symptomatic hypoglycemia (with blood glucose $\leq 3.9 \mathrm{mmol} / \mathrm{L}(\leq 70 \mathrm{mg} / \mathrm{dL})$ ).

Safety endpoints included incidence of documented symptomatic hypoglycemia and gastrointestinal (GI) adverse events (nausea, vomiting, and diarrhea).

\section{Patient involvement}

Patients were not involved in this data analysis.

\section{Statistical analysis}

Comparisons for participants from NA versus RoW included: all patients with baseline and on-study (last observation carried forward) assessments for HbAlc, PPG, FPG, and weight using two sample t-tests; all patients with follow-up assessments for response and adverse event (AE) rates using $\chi^{2}$ tests. For the iGlarLixi and iGlar treatment arms in the two studies the smallest number of NA patients was 91. For comparisons between NA and RoW subgroups within the iGlarLixi and iGlar treatment arms, with the observed sample sizes in the two studies, there was $80 \%-95 \%$ power for effect sizes (differences in means/SD) of $\geq 0.35$ and for differences in rates of $0.12-0.17$ depending on rates, ranging from 0.05 to 0.5 , respectively.

Comparisons between treatment arms within NA and RoW subgroups were carried out using two-factor analysis of variance (ANOVA) for continuous endpoints and $\chi^{2}$ tests or Fisher's exact tests for discrete outcomes.

\section{RESULTS}

\section{Patients}

Analysis included 1167 patients from LixiLan-O (NA, $\mathrm{n}=371$; RoW, $\mathrm{n}=796$ ) and 731 from LixiLan-L (NA, $\mathrm{n}=196$; RoW, $\mathrm{n}=535$ ). Baseline demographics are summarised in table 1 . In both studies, the NA patient groups included a significantly higher proportion of Black $(\mathrm{p}<0.001)$ and Asian patients $(p<0.001)$ versus the RoW group. In LixiLan-O, the duration of T2D was significantly longer in the NA versus the RoW group (10.0 vs 8.2 years, respectively; $\mathrm{p}<0.001)$.

\section{Efficacy}

In LixiLan-O, NA patients had significantly higher baseline HbA1c versus RoW patients across all treatment groups; there was no significant difference in baseline HbA1c in LixiLan-L (table 2). No significant difference was observed between NA and RoW patients in mean HbA1c change from baseline to Week 30 across all treatment groups in either study (table 2).

Treatment with iGlarLixi resulted in significantly greater reductions in $\mathrm{HbAlc}$ versus either iGlar or lixisenatide alone in both NA and RoW patients in LixiLan-O (all $\mathrm{p} \leq 0.003$ ) and LixiLan-L (all $\mathrm{p}<0.001)$ (table 3). A significantly higher proportion of patients in the RoW group achieved HbA1c $<42 \mathrm{mmol} / \mathrm{mol}(<7.0 \%)$ across all treatment groups in LixiLan-O, and within the iGlarLixi group in LixiLan-L (figure 1A,B). Treatment with iGlarLixi resulted in a significantly greater proportion of patients achieving HbAlc $<42 \mathrm{mmol} / \mathrm{mol}(<7.0 \%)$ versus either iGlar or lixisenatide alone in both patient groups across both studies (figure 1A,B).

Mean baseline PPG levels were significantly lower in NA patients in all treatment groups, with the exception of the lixisenatide group in LixiLan-O (table 2). There was no significant difference in change in PPG level from baseline to Week 30 between patient groups, within the studies (table 2). Treatment with iGlarLixi resulted in significantly greater reductions in PPG versus either iGlar or lixisenatide alone in both the NA and RoW patient groups in LixiLan-O (all $\mathrm{p} \leq 0.007$ ) and LixiLan-L (all $\mathrm{p}<0.001$ ) (table 3).

\begin{tabular}{|c|c|c|c|c|c|c|}
\hline & \multicolumn{3}{|l|}{ LixiLan-O } & \multicolumn{3}{|l|}{ LixiLan-L } \\
\hline & $\begin{array}{l}\text { NA } \\
n=371\end{array}$ & $\begin{array}{l}\text { RoW } \\
n=796\end{array}$ & $P$ value & $\begin{array}{l}\text { NA } \\
n=196\end{array}$ & $\begin{array}{l}\text { RoW } \\
\mathrm{n}=535\end{array}$ & $P$ value \\
\hline Age, years & $57.9(10.1)$ & $58.6(8.9)$ & 0.246 & $59.9(9.2)$ & $60.0(9.0)$ & 0.932 \\
\hline Women, \% & $45.8(49.9)$ & $51.0(50.0)$ & 0.098 & $48.0(50.1)$ & 55.5 (49.7) & 0.070 \\
\hline BMI, $\mathrm{kg} / \mathrm{m}^{2}$ & $31.6(4.7)$ & $31.8(4.3)$ & 0.402 & $30.9(4.3)$ & $31.2(4.1)$ & 0.264 \\
\hline \multicolumn{7}{|l|}{ Race, \% } \\
\hline White & 81.1 (39.2) & $94.2(23.3)$ & $<0.001$ & $73.0(44.5)$ & $98.5(12.1)$ & $<0.001$ \\
\hline Black & $13.5(34.2)$ & 3.5 (18.4) & $<0.001$ & $18.9(39.2)$ & $0.2(4.3)$ & $<0.001$ \\
\hline Asian & $4.3(20.3)$ & $0.3(5.0)$ & $<0.001$ & $8.2(27.5)$ & $0.7(8.6)$ & $<0.001$ \\
\hline Other & $1.1(10.3)$ & $2.0(14.0)$ & 0.203 & $0.0(0.0)$ & $0.6(7.5)$ & 0.082 \\
\hline T2D duration, years & $10.0(6.3)$ & $8.2(5.3)$ & $<0.001$ & $12.5(7.0)$ & $11.9(6.6)$ & 0.257 \\
\hline OADs, $\mathrm{n}$ & $1.5(0.5)$ & $1.6(0.5)$ & $<0.001$ & $1.3(0.6)$ & $1.4(0.6)$ & 0.477 \\
\hline Insulin dose, units & $36.9(8.6)$ & $34.4(8.9)$ & 0.001 & $45.5(12.1)$ & 46.4 (12.9) & 0.410 \\
\hline
\end{tabular}

Data are presented as the mean (SD) or as indicated.

BMI, body mass index; NA, North America; OADs, oral antidiabetic drugs; RoW, rest of the world; SD, standard deviation; T2D, type 2 diabetes. 
Table 2 Baseline and change from baseline at Week 30 in glycemic parameters and body weight, and achievement of composite endpoint, for North American and RoW patients in LixiLan-O (A) and LixiLan-L (B)

\begin{tabular}{|c|c|c|c|c|c|c|c|c|c|}
\hline & \multicolumn{3}{|l|}{ iGlarLixi } & \multicolumn{3}{|l|}{ iGlar } & \multicolumn{3}{|l|}{ Lixisenatide } \\
\hline & $\begin{array}{l}\text { NA } \\
n=136\end{array}$ & $\begin{array}{l}\text { RoW } \\
n=332\end{array}$ & $\begin{array}{l}P \\
\text { value }\end{array}$ & $\begin{array}{l}\text { NA } \\
n=155\end{array}$ & $\begin{array}{l}\text { RoW } \\
n=311\end{array}$ & $\begin{array}{l}P \\
\text { value }\end{array}$ & $\begin{array}{l}\text { NA } \\
n=80\end{array}$ & $\begin{array}{l}\text { RoW } \\
n=153\end{array}$ & $\begin{array}{l}P \\
\text { value }\end{array}$ \\
\hline \multicolumn{10}{|l|}{$\mathrm{HbA} 1 \mathrm{c}, \mathrm{mmol} / \mathrm{mol}$} \\
\hline Baseline & $89.5(7.5)$ & $87.8(7.8)$ & 0.031 & $89.8(7.8)$ & $87.6(7.3)$ & 0.002 & $91.2(8.5)$ & $87.7(7.3)$ & 0.001 \\
\hline Change from baseline & $-16.7(9.9)$ & $-16.7(9.5)$ & 0.995 & $-13.3(9.3)$ & $-13.6(9.6)$ & 0.805 & $-7.7(9.7)$ & $-9.2(9.4)$ & 0.221 \\
\hline \multicolumn{10}{|l|}{$\mathrm{HbA} 1 \mathrm{c}, \%$} \\
\hline Baseline & $8.19(0.69)$ & $8.03(0.71)$ & 0.031 & $8.22(0.71)$ & $8.01(0.67)$ & 0.002 & $8.34(0.78)$ & $8.02(0.67)$ & 0.001 \\
\hline Change from baseline & $-1.53(0.91)$ & $-1.53(0.87)$ & 0.995 & $-1.22(0.85)$ & $-1.24(0.88)$ & 0.805 & $-0.70(0.89)$ & $-0.84(0.86)$ & 0.221 \\
\hline $\mathrm{PPG},{ }^{\star} \mathrm{mmol} / \mathrm{L}$ & $n=115$ & $\mathrm{n}=315$ & & $n=132$ & $n=298$ & & $n=62$ & $n=134$ & \\
\hline Baseline & $14.60(3.71)$ & $15.41(3.58)$ & 0.040 & $13.88(3.27)$ & $14.93(3.75)$ & 0.005 & $14.53(3.09)$ & $14.81(3.43)$ & 0.584 \\
\hline Change from baseline & $-6.45(4.40)$ & $-5.90(4.22)$ & 0.237 & $-3.37(3.43)$ & $-3.22(3.59)$ & 0.690 & $-4.60(3.94)$ & $-4.79(4.20)$ & 0.753 \\
\hline $\mathrm{FPG}, \mathrm{mmol} / \mathrm{L}$ & & & & & & & & & \\
\hline Baseline & 10.07 (2.39) & $9.79(2.33)$ & 0.244 & $9.76(2.41)$ & $9.75(2.29)$ & 0.948 & $9.85(2.24)$ & $9.76(2.13)$ & 0.764 \\
\hline Change from baseline & $-3.63(2.59)$ & $-3.37(2.58)$ & 0.309 & $-3.06(2.86)$ & $-3.18(2.72)$ & 0.673 & $-1.81(2.95)$ & $-1.22(2.45)$ & 0.105 \\
\hline Weight† (kg) & & & & & & & & & \\
\hline Baseline & $91.8(18.0)$ & $88.5(16.8)$ & 0.055 & $89.8(16.8)$ & $89.7(16.2)$ & 0.944 & $89.5(18.2)$ & $91.5(15.2)$ & 0.378 \\
\hline Change from baseline & $0.3(4.2)$ & $-0.5(3.4)$ & 0.034 & $1.8(4.1)$ & $0.75(4.1)$ & 0.008 & $-1.48(3.2)$ & $-2.74(3.4)$ & 0.006 \\
\hline Insulin dose (units) Week 30 & $40.6(14.7)$ & $38.3(15.2)$ & 0.128 & $40.6(14.6)$ & $39.2(15.4)$ & 0.342 & $\mathrm{~N} / \mathrm{A}$ & $\mathrm{N} / \mathrm{A}$ & $\mathrm{N} / \mathrm{A}$ \\
\hline Composite endpointł, n (\%) & $35(25.7)$ & $114(34.3)$ & 0.060 & $17(11.0)$ & $71(22.8)$ & 0.001 & $11(13.8)$ & $50(32.7)$ & $<0.001$ \\
\hline & iGlarLixi & & & & iGlar & & & & \\
\hline & $\begin{array}{l}\text { NA } \\
n=105\end{array}$ & $\begin{array}{l}\text { RoW } \\
n=261\end{array}$ & & $P$ value & $\begin{array}{l}\text { NA } \\
n=91\end{array}$ & & $\begin{array}{l}\text { RoW } \\
n=274\end{array}$ & P va & lue \\
\hline $\mathrm{HbA} 1 \mathrm{c}, \mathrm{mmol} / \mathrm{mol}$ & & & & & & & & & \\
\hline Baseline & $88.8(7.8)$ & 88.0( & 7.3) & 0.319 & $88.8(8.0$ & & $88.1(8.0)$ & 0.5 & \\
\hline Change from baseline & $-11.8(11.1)$ & -12.0 & $(9.2)$ & 0.836 & $-6.2(9.2$ & & $-6.4(9.5)$ & 0.8 & \\
\hline $\mathrm{HbA} 1 \mathrm{c}, \%$ & & & & & & & & & \\
\hline Baseline & $8.12(0.71)$ & 8.05 & $0.67)$ & 0.319 & $8.12(0.7$ & & $8.06(0.73)$ & 0.5 & \\
\hline Change from baseline & $-1.08(1.01)$ & -1.10 & $(0.84)$ & 0.836 & $-0.57(0$ & $.84)$ & $-0.59(0.87)$ & 0.8 & \\
\hline $\mathrm{PPG},{ }^{*} \mathrm{mmol} / \mathrm{L}$ & $n=86$ & $\mathrm{n}=246$ & & & $n=81$ & & $n=259$ & & \\
\hline Baseline & $13.78(3.29)$ & 15.23 & (3.92) & 0.002 & 13.59 & $.97)$ & $15.40(3.47)$ & $<0.0$ & \\
\hline Change from baseline & $-4.50(4.51)$ & -5.10 & $(4.48)$ & 0.286 & $-1.09(5$ & 18) & $-1.70(3.85)$ & 0.2 & 54 \\
\hline $\mathrm{FPG}, \mathrm{mmol} / \mathrm{L}$ & & & & & & & & & \\
\hline Baseline & $6.91(2.04)$ & 7.49 & (1.88) & 0.009 & $6.90(2.0$ & & $7.46(2.08)$ & 0.0 & \\
\hline Change from baseline & $-0.38(2.92)$ & -0.49 & (2.59) & 0.715 & $-0.09(3$ & .05) & $-0.77(2.56)$ & 0.0 & 36 \\
\hline Weight, kg & & & & & & & & & \\
\hline Baseline & $87.6(14.0)$ & 87.9 & 14.6) & 0.832 & $89.7(16$ & & $86.2(14.2)$ & 0.0 & \\
\hline Change from baseline & $-0.4(3.8)$ & -0.6( & 3.0) & 0.710 & $1.2(2.9)$ & & $0.7(2.7)$ & 0.1 & \\
\hline Insulin dose, units & & & & & & & & & \\
\hline Baseline & $36.3(9.1)$ & 34.43 & $(9.2)$ & 0.086 & $37.62(8$ & & $34.43(8.7)$ & 0.0 & \\
\hline Change from baseline & $8.7(11.5)$ & 11.49 & $(10.2)$ & 0.025 & $8.77(9.5$ & & $12.37(9.9)$ & 0.0 & \\
\hline Composite endpoint $\neq, n$ (\%) & $14(13.3)$ & $59(22$ & & 0.028 & $9(9.9)$ & & $24(8.8)$ & 0.7 & \\
\hline
\end{tabular}

Data are mean (SD).

*Measured 2 hours after standard liquid meal in a subset of patients.

†iGlarLixi arm: NA, $n=135$; RoW, $n=332$.

$\ddagger$ Composite endpoint of $\mathrm{HbA} 1 \mathrm{c}<42 \mathrm{mmol} / \mathrm{mol}(<7.0 \%)$, no weight gain or symptomatic hypoglycemia (blood glucose $\leq 3.9 \mathrm{mmol} / \mathrm{L}(\leq 70 \mathrm{mg} / \mathrm{dL})$ ).

FPG, fasting plasma glucose; iGlar, insulin glargine; iGlarLixi, insulin glargine/lixisenatide; NA, North America; PPG, postprandial plasma glucose;

RoW, rest of the world. 
Table 3 Difference in change in glycemic parameters and body weight from baseline to Week 30 for NA and RoW patients by treatment group in LixiLan-O (A) and LixiLan-L (B)

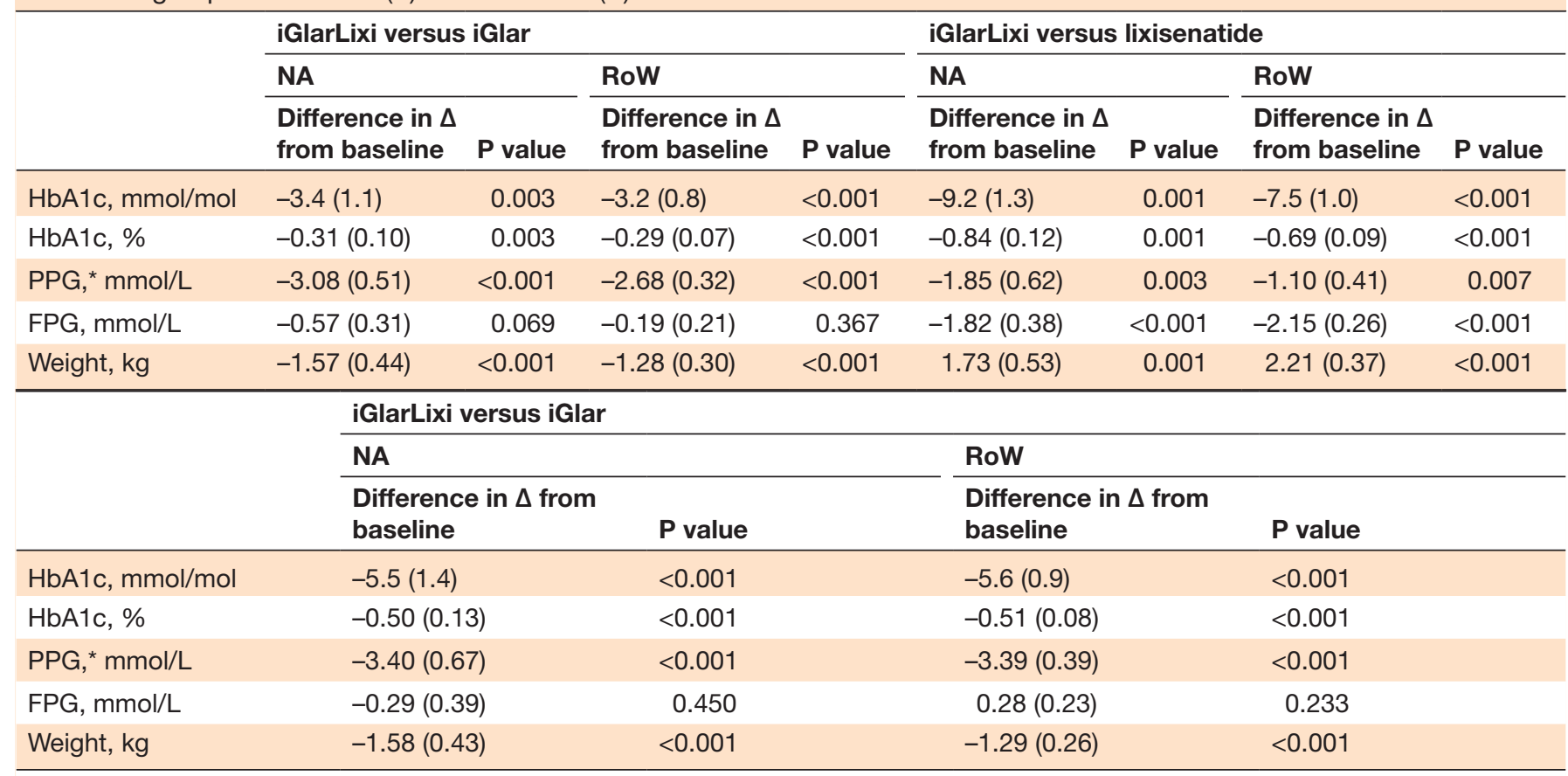

Data are mean (SE).

*Measured 2 hours after standard liquid meal in a subset of patients.

FPG, fasting plasma glucose; iGlar, insulin glargine; iGlarLixi, insulin glargine/lixisenatide; NA, North America; PPG, postprandial plasma glucose; RoW, rest of the world.

There were no significant differences in mean baseline FPG levels in both patient groups in LixiLan-O, but they were significantly lower in NA in LixiLan-L (table 2). Generally, there was no significant difference in change in FPG from baseline to Week 30 between NA and RoW patients within treatment groups, with the exception of the iGlar group in LixiLan-L (table 2). In Lixilan-O, both NA and RoW patients showed greater FPG reduction with iGlarLixi versus lixisenatide alone $(\mathrm{p}<0.001)$ (table 3$)$. In Lixilan-L, there was no significant difference in change from baseline to Week 30 in mean FPG between treatment groups (table 3 ).

\section{Insulin dose}

In LixiLan-L, in the iGlarLixi-treated group, baseline insulin doses for NA and RoW patients were similar ( $36.28 \mathrm{U} /$ day and $34.43 \mathrm{U} /$ day, respectively; $\mathrm{p}=0.086$ ). In the iGlar-treated group, doses were significantly higher in the NA group versus the RoW group (37.62 U/day and
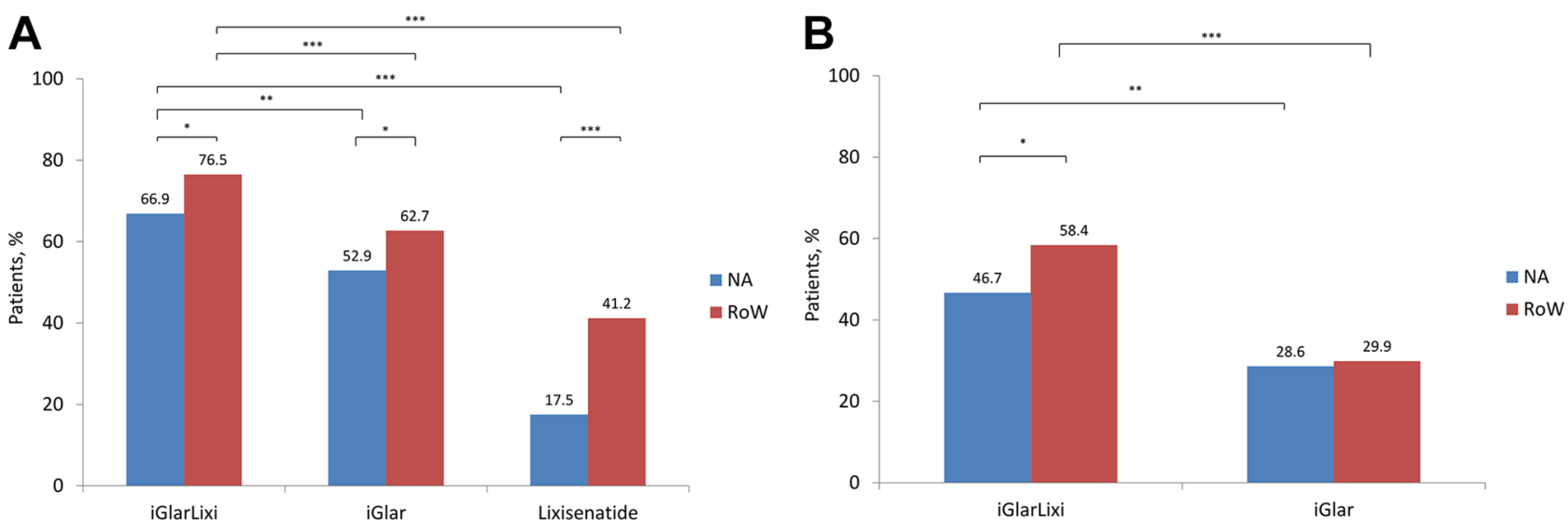

*P $\leq 0.05 ; * * P \leq 0.01 ; * * * P \leq 0.001$

${ }^{*} \mathrm{P} \leq 0.05 ; * * \mathrm{P} \leq 0.01 ; * * * \mathrm{P} \leq 0.001$

Figure 1 Proportion of NA and RoW patients achieving HbA1c $<7.0 \%$ in (A) LixiLan-O and (B) LixiLan-L. iGlar, insulin glargine; iGlarLixi, insulin glargine/lixisenatide; NA, North America; RoW, rest of the world. 
$34.43 \mathrm{U} /$ day, respectively; $\mathrm{p}=0.002$ ). At the end of the study period, in the iGlarLixi-treated group, the increase in insulin dose was significantly lower in NA patients (8.72 $\mathrm{U} /$ day vs $11.49 \mathrm{U} /$ day; $\mathrm{p}=0.025)$. A similar result was seen in the iGlar-treated group (NA: $8.77 \mathrm{U} /$ day vs RoW: $12.37 \mathrm{U} /$ day; $\mathrm{p}=0.003$ ).

\section{Weight change}

No significant differences were observed in mean body weight at baseline between NA and RoW patients within treatment groups in either study (table 2). Interestingly, in LixiLan-O, NA patients treated with iGlarLixi showed a small increase in mean weight from baseline, while RoW patients showed a small reduction. Weight gain was slightly greater in NA patients treated with iGlar, and weight loss was lower in NA patients treated with lixisenatide versus RoW patients. In LixiLan-L, iGlarLixi was associated with a small magnitude of weight loss in both NA and RoW patients (with no significant differences between regions) versus patients treated with iGlar, who had weight gain in both groups (table 2). There were significant differences in weight change between treatment arms in both studies-iGlarLixi had a beneficial weight profile versus iGlar (table 3 ).

\section{Hypoglycemia}

No significant differences were observed between NA and RoW in the proportion of patients experiencing documented symptomatic hypoglycemia (blood glucose $<3.9 \mathrm{mmol} / \mathrm{L}(70 \mathrm{mg} / \mathrm{dL}))$, in any treatment group, in either study (figure 2). Rates of documented symptomatic hypoglycemia with iGlarLixi were numerically lower versus iGlar in both NA and RoW patients in LixiLan-L and in NA patients in LixiLan-O, but these differences did not reach statistical significance (figure 2). Rates of documented symptomatic hypoglycemia were significantly higher in patients on iGlarLixi versus those on lixisenatide (LixiLan-O), regardless of region.

\section{Gastrointestinal adverse events}

In general, NA patients tended to report higher rates of adverse events, and this reached significance for nausea in the LixiLan-O study, in which the incidence was significantly higher in NA versus RoW patients treated with iGlar $(7.1 \%$ vs $1.9 \% ; \mathrm{p}=0.020)$ and lixisenatide $(33.8 \%$ vs $19.0 \% ; \mathrm{p}=0.016)$, but not iGlarLixi (9.6\% vs $9.6 \%$; $\mathrm{p}=0.986$ ) (table 4). Overall, treatment with iGlarLixi was associated with a significantly lower incidence of nausea versus lixisenatide in both NA $(9.6 \%$ vs $33.8 \%$; $\mathrm{p}<0.001)$ and RoW patients $(9.6 \%$ vs $19.0 \%$; $=0.009)$ in LixiLan-O.

\section{Composite endpoint}

NA patients tended to be less likely than RoW patients to achieve the composite endpoint of HbA1c <42 mmol/ mol $(<7.0 \%)$, no weight gain or symptomatic hypoglycemia (blood glucose $\leq 3.9 \mathrm{mmol} / \mathrm{L} \quad(\leq 70 \mathrm{mg} / \mathrm{dL})$ ). This reached statistical significance in the iGlar $(11.0 \%$ vs $22.8 \% ; \mathrm{p}=0.001)$ and lixisenatide $(13.8 \%$ vs $32.7 \%$; $\mathrm{p}<0.001)$ treatment groups of LixiLan-O (table 2A) and the iGlarLixi $(13.3 \%$ vs $22.6 \%$; $\mathrm{p}=0.028)$ treatment group of LixiLan-L (table 2B).

\section{DISCUSSION}

In this post hoc analysis of the LixiLan-O and LixiLan-L RCTs, we observed that the glucose-lowering efficacy of the titratable fixed-ratio combination of basal insulin glargine (U100) and the GLP-1 RA lixisenatide, iGlarLixi, was similar in NA and RoW patients with T2D. The beneficial effect on weight for iGlarLixi, compared with iGlar, was also consistent across geographic regions. Furthermore, geographic region did not significantly impact hypoglycemia risk or GI adverse events, despite differences in baseline characteristics.

Diabetes is a truly international health challenge, with prevalence expected to increase in all areas of the world over the coming years. ${ }^{21}$ Previous studies have reported differing effects of geographic region on outcomes of therapy. ${ }^{4522}$ Being a large and growing global problem,
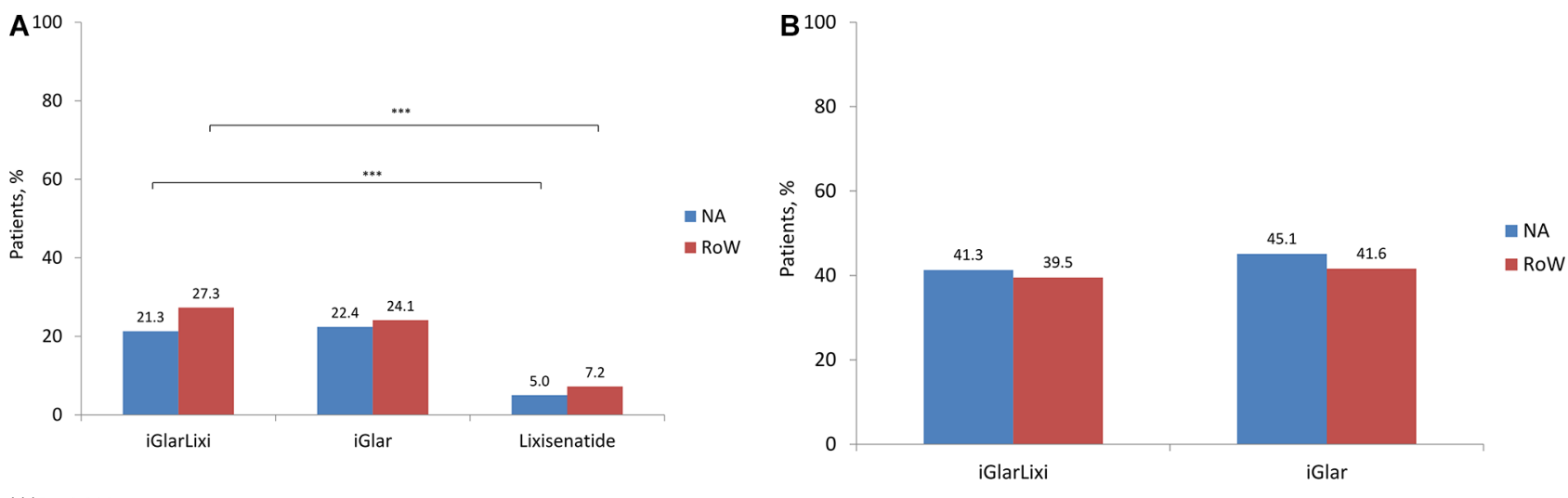

$* * * P \leq 0.001$.

Figure 2 Proportion of patients experiencing documented symptomatic hypoglycemia (blood glucose $<3.9 \mathrm{mmol} / \mathrm{L}$ ( $70 \mathrm{mg} /$ $\mathrm{dL})$ ) in NA and RoW patients in (A) LixiLan-O and (B) LixiLan-L. iGlar, insulin glargine; iGlarLixi, insulin glargine/lixisenatide; NA, North America; RoW, rest of the world. 
Table 4 Nausea, vomiting, and diarrhea in North American and RoW patients in (A) LixiLan-O and (B) LixiLan-L

\begin{tabular}{|c|c|c|c|c|c|c|c|c|c|}
\hline \multirow[b]{2}{*}{ LixiLan-O } & \multicolumn{3}{|l|}{ iGlarLixi } & \multicolumn{3}{|l|}{ iGlar } & \multicolumn{3}{|c|}{ Lixisenatide } \\
\hline & $\begin{array}{l}\text { NA } \\
n=136\end{array}$ & $\begin{array}{l}\text { Row } \\
n=333\end{array}$ & $P$ value & $\begin{array}{l}\text { NA } \\
n=156\end{array}$ & $\begin{array}{l}\text { Row } \\
n=311\end{array}$ & $P$ value & $\begin{array}{l}\text { NA } \\
n=80\end{array}$ & $\begin{array}{l}\text { RoW } \\
n=153\end{array}$ & P value \\
\hline Nausea & $13(9.6)$ & 32 (9.6) & 0.986 & $11(7.1)$ & $6(1.9)$ & 0.020 & 27 (33.8) & 29 (19.0) & 0.016 \\
\hline Vomiting & $7(5.1)$ & $8(2.4)$ & 0.185 & $4(2.6)$ & $3(1.0)$ & 0.247 & 9 (11.3) & $6(3.9)$ & 0.058 \\
\hline \multirow[t]{2}{*}{ Diarrhea } & 14 (10.3) & $28(8.4)$ & 0.532 & $9(5.8)$ & $11(3.5)$ & 0.297 & $12(15.0)$ & $9(5.9)$ & 0.039 \\
\hline & \multicolumn{2}{|c|}{ iGlarLixi } & & & & \multicolumn{2}{|l|}{ iGlar } & & \\
\hline LixiLan-L & \multicolumn{2}{|c|}{$\begin{array}{l}\text { NA } \\
n=104\end{array}$} & $\begin{array}{l}\text { RoW } \\
n=261\end{array}$ & P value & & $\begin{array}{l}\text { NA } \\
n=91\end{array}$ & $\begin{array}{l}\text { RoW } \\
n=274\end{array}$ & \multicolumn{2}{|c|}{$P$ value } \\
\hline Nausea & \multicolumn{2}{|c|}{14 (13.5) } & $24(9.2)$ & 0.261 & & $1(1.1)$ & $1(0.4)$ & \multicolumn{2}{|c|}{0.524} \\
\hline Vomiting & \multicolumn{2}{|c|}{$6(5.8)$} & $7(2.7)$ & 0.216 & & 0 & $2(0.7)$ & \multicolumn{2}{|c|}{0.156} \\
\hline Diarrhea & \multicolumn{2}{|c|}{$5(4.8)$} & $11(4.2)$ & 0.808 & & $5(5.5)$ & $5(1.8)$ & \multicolumn{2}{|c|}{0.146} \\
\hline
\end{tabular}

Data are $n,(\%)$.

iGlar, insulin glargine; iGlarLixi, insulin glargine/lixisenatide; NA, North America; RoW, rest of the world.

it is important to know whether efficacy and safety of antihyperglycemic medications differ between regions, relevant to trial considerations and reporting of trial results. To date, studies in diabetes have been mixed. For example, in the Action in Diabetes and Vascular Disease: Preterax and Diamicron MR Controlled Evaluation trial (ADVANCE), despite differences in baseline characteristics, geographic region (Asia, established market economies, and Eastern Europe) had no impact on the effects of intensive glycemic control. ${ }^{22}$ In contrast, a meta-analysis of clinical trials comparing intensive treatment with standard of care for glycemic control in T2D conducted in NA and RoW patients found differences between regions, with intensive therapy being associated with increased severe hypoglycemia, all-cause mortality, and cardiovascular mortality in NA versus RoW patients. ${ }^{4}$

Similarities in change from baseline in HbA1c, PPG and FPG between NA and RoW participants in the current study were seen despite differences in baseline characteristics. Baseline differences, such as diabetes duration, age of onset and race, which are generally associated with poorer outcomes, were observed in a significantly higher proportion of participants in the NA group versus the RoW group. Additionally, a greater proportion of RoW participants achieved an HbAlc target of $<7 \%(<42$ $\mathrm{mmol} / \mathrm{mol}$ ) at the end of study periods. Particularly in LixiLan-O, RoW patients were more likely to achieve the composite endpoint of $\mathrm{HbA1c}<7 \%$ with no weight gain and no documented hypoglycemia than NA patients in all treatment groups. Taking into consideration that the absolute change from baseline in $\mathrm{HbAlc}$ and incidence rate of hypoglycemia during the studies were not significantly different between both groups suggests that this discrepancy in target achievement may reflect the higher baseline HbAlc and less favorable effect on weight seen in NA versus RoW patients.

Notably, in both NA and RoW patients in our analysis, the rate of GI adverse events was lower in patients treated with iGlarLixi versus lixisenatide in LixiLan-O, likely as a result of the gradual titration of the lixisenatide dose parallel to the iGlar titration with fixed-ratio iGlarLixi versus the separate, fixed-dose administration of lixisenatide alone. Overall, NA patients reported more adverse events in all treatment groups, but the overall trends for individual adverse events were similar. Thus, the enhanced safety profile of iGlarLixi, demonstrated both in NA and RoW regions of the world, should have important clinical implications.

Diabetes treatment guidelines focus on a patient-centered approach to guide the choice of pharmacological agents, with consideration being given to efficacy with structured recommendations for patients with cardiovascular disease, cost, potential side effects, weight, co-morbidities, hypoglycemia risk and patient preferences such as simplified treatment administration. ${ }^{67}$ The use of combination therapy with agents that act on different aspects of the pathophysiology of T2D with complementary mechanisms of action is in accordance with the suggested treatment approach for the management of T2D ${ }^{1023}$ iGlarLixi combines iGlar, which acts predominantly on FPG levels, with a short-acting GLP-1 RA that slows gastric emptying and has a glucose-dependent action predominantly on PPG levels by enhancing glucose-dependent insulin secretion by $\beta$-cells, decreasing glucagon secretion by $\alpha$-cells, and increasing satiety. ${ }^{16}$ Understanding the mechanism of action of the individual components and potential synergies of the combination helps to explain the findings of our study, which demonstrated greater reductions in PPG, as well as HbA1c, with iGlarLixi versus both iGlar and lixisenatide alone, among both regions.

Management of T2D largely focuses on the assessment of optimal glycemic control using HbAlc. However, increasing evidence suggests that elevated PPG is also linked to the risk of diabetes complications, and may be a better predictor of cardiovascular and all-cause mortality versus FPG. ${ }^{724}$ The indisputable value of tighter PPG control and glucose variability has not been proven, largely due to a lack of well-designed, long-term outcome 
studies assessing PPG as the primary target. ${ }^{7}$ Among patients on insulin treatment, most treatment guidelines do not specify a preference for any particular combination therapies, but based on recent data, the 2018 update of the American Diabetes Association/European Association for the Study of Diabetes recommended adding a GLP-1 RA to intensify basal insulin therapy. ${ }^{6}$ In addition, the LixiLan-O trial demonstrated glycemic and GI tolerability benefits and mitigation of the weight effects of insulin in these insulin-naive patients with uncontrolled T2D treated with iGlarLixi over basal insulin or lixisenatide alone, which suggests it may be useful to reassess the currently recommended treatment approach of sequential addition of injectable therapies toward the use of a titratable fixed-ratio combination. ${ }^{19}$

This study has the limitation of its post hoc design and the relatively short treatment duration. In addition, data from randomized, clinical trials with highly controlled patient selection may not be fully generalizable to realworld populations. Additionally, generalizability of the present study may also be limited by non-participation of Asian or African countries in LixiLan-O and LixiLan-L. It is also possible that there are differences in response between ethnicities, as has been seen in other studies. For example, though it would be desirable to compare Caucasian and African American ethnicities in the NA analysis in our study, the number of African American patients was too low for a valid comparison to be made. Further studies are in development to investigate the efficacy and safety of iGlarLixi in Asian, African American and Hispanic patients with T2D. Nevertheless, the results are reassuring in that iGlarLixi was similarly effective in both NA and RoW patients studied, irrespective of differences in baseline characteristics.

\section{CONCLUSIONS}

This post hoc analysis of the LixiLan-O and LixiLan-L studies demonstrated that glucose-lowering efficacy with iGlarLixi was generally similar in patients with T2D in NA and RoW, with no significant differences in hypoglycemia risk or GI adverse events, despite baseline differences in patient characteristics between NA and RoW. These data add to the body of information on treatment outcomes with iGlarLixi, specifically in the North American population.

Acknowledgements The authors would like to thank Dr Timothy Bailey of AMCR Institute, Inc., Escondido, California, USA, for his input during earlier stages of data analysis for this publication. The authors received writing/editorial support in the preparation of this manuscript provided by Rasilaben Vaghjiani, PhD, and Michael van der Veer, PhD, of Excerpta Medica, funded by Sanofi US, Inc.

Contributors All authors contributed equally to the manuscript. Data were analyzed by BS

Funding This study was funded by Sanofi US, Inc. The sponsors, or its employees, were involved in the study design, analysis and interpretation of the data and in the decision to submit for publication. The authors received writing/editorial support in the preparation of this manuscript provided by Rasilaben Vaghijiani, $\mathrm{PhD}$, and Michael van der Veer, PhD, of Excerpta Medica, funded by Sanofi US, Inc.
Competing interests GD: advisory panel and consultant for Sanofi; research support received from Lexicon, Lilly, Mylan, Novo Nordisk, and Sanofi; speakers' bureaus for AstraZeneca and Sanofi. HSB: Speakers' bureaus for and received honoraria from Abbott, Amgen, AstraZeneca, Bayer, Boehringer Ingelheim, Janssen, Lilly, Merck \& Co., Mylan, Novo Nordisk, sanofi-aventis, and Valeant; clinical research for AstraZeneca, Boehringer Ingelheim, Genkyotex, Janssen, Lilly, Merck \& Co., Pfizer, Novo Nordisk, Regeneron, sanofi-aventis, and Valeant. TD: employee of Sanofi US, Inc. and stockholder of Sanofi. MG: employee of Sanofi Canada. WS: employee of Sanofi US, Inc. and stockholder of Sanofi. AV: board member for Medscape; consultant for Alnylam, Astellas, Hydra Biosciences, ISIS Pharmaceuticals, Merck \& Co., Mitsubishi Tanabe, Neurometrix, Pamlab, and Pfizer research support from ADA, Daiichi Sankyo, Impeto Medical, Intarcia, NIH, Novo Nordisk, Pfizer, Tercica, VeroScience, and ViroMed; speakers' bureaus for Merck \& Co. and Pamlab.

\section{Patient consent for publication Not required.}

Provenance and peer review Not commissioned; externally peer reviewed.

\section{Data sharing statement № additional data are available.}

Open access This is an open access article distributed in accordance with the Creative Commons Attribution Non Commercial (CC BY-NC 4.0) license, which permits others to distribute, remix, adapt, build upon this work non-commercially, and license their derivative works on different terms, provided the original work is properly cited, appropriate credit is given, any changes made indicated, and the use is non-commercial. See: http://creativecommons.org/licenses/by-nc/4.0/.

\section{REFERENCES}

1. Hu FB. Globalization of diabetes: the role of diet, lifestyle, and genes. Diabetes Care 2011;34:1249-57.

2. Ji L, Min KW, Oliveira J, et al. Comparison of efficacy and safety of two starting insulin regimens in non-Asian, Asian Indian, and East Asian patients with type 2 diabetes: a post hoc analysis of the PARADIGM study. Diabetes Metab Syndr Obes 2016;9:243-9.

3. Kirk JK, D'Agostino RB, Bell RA, et al. Disparities in HbA1c levels between African-American and non-Hispanic white adults with diabetes: a meta-analysis. Diabetes Care 2006;29:2130-6.

4. Sardar P, Udell JA, Chatterjee S, et al. Effect of intensive versus standard blood glucose control in patients with type 2 diabetes mellitus in different regions of the world: systematic review and meta-analysis of randomized controlled trials. J Am Heart Assoc 2015;4:e001577.

5. Rutten GE, Tack CJ, Pieber TR, et al. LEADER 7: cardiovascular risk profiles of US and European participants in the LEADER diabetes trial differ. Diabetol Metab Syndr 2016;8:37.

6. American Diabetes Association. Standards of medical care in diabetes-2017. Diabetes Care 2017;40(Suppl. 1):S1-S142.

7. Cheng AYY, Canadian Diabetes Association Clinical Practice Guidelines Expert Committee. Canadian diabetes association 2013 clinical practice guidelines for the prevention and management of diabetes in Canada. Introduction. Can J Diabetes 2013;37(Suppl 1):S1-3. [Epub ahead of print].

8. Garber AJ, Abrahamson MJ, Barzilay Jl, et al. Consensus Statement by the American Association of Clinical Endocrinologists and American College of Endocrinology on the Comprehensive Type 2 Diabetes Management Algorithm - 2017 Executive Summary. Endocr Pract 2017;23:207-38.

9. Davies MJ, D'Alessio DA, Fradkin J, et al. Management of Hyperglycemia in Type 2 Diabetes, 2018. A Consensus Report by the American Diabetes Association (ADA) and the European Association for the Study of Diabetes (EASD). Diabetes Care 2018:41:2669-701.

10. Defronzo RA. Banting Lecture. From the triumvirate to the ominous octet: a new paradigm for the treatment of type 2 diabetes mellitus. Diabetes 2009;58:773-95.

11. Diamant M, Nauck MA, Shaginian R, et al. Glucagon-like peptide 1 receptor agonist or bolus insulin with optimized basal insulin in type 2 diabetes. Diabetes Care 2014:37:2763-73.

12. Dalal MR, Xie L, Baser O, et al. Adding rapid-acting insulin or GLP-1 receptor agonist to basal insulin: outcomes in a community setting. Endocr Pract 2015;21:68-76.

13. Rosenstock J, Fonseca VA, Gross JL, et al. Advancing basal insulin replacement in type 2 diabetes inadequately controlled with insulin glargine plus oral agents: a comparison of adding albiglutide, a weekly GLP-1 receptor agonist, versus thrice-daily prandial insulin lispro. Diabetes Care 2014;37:2317-25.

14. Rosenstock J, Schwartz SL, Clark CM, et al. Basal insulin therapy in type 2 diabetes: 28-week comparison of insulin glargine (HOE 901) and NPH insulin. Diabetes Care 2001;24:631-6. 
15. Becker RH, Stechl J, Steinstraesser A, et al. Lixisenatide reduces postprandial hyperglycaemia via gastrostatic and insulinotropic effects. Diabetes Metab Res Rev 2015;31:610-8.

16. Fineman MS, Cirincione BB, Maggs D, et al. GLP-1 based therapies: differential effects on fasting and postprandial glucose. Diabetes Obes Metab 2012;14:675-88.

17. FDA, 2016. Center for Drug Evaluation and Research, Application number: 208471Orig1s000 [Adlyxin/lixisenatide]. Summary Review. Available: http://www.accessdata.fda.gov/drugsatfda_docs/nda/ 2016/208471Orig1s000SumR.pdf [Accessed 30 January 2019].

18. Aroda VR, Rosenstock J, Wysham C, et al. Efficacy and safety of LixiLan, a titratable fixed-ratio combination of insulin glargine plus lixisenatide in type 2 diabetes inadequately controlled on basa insulin and metformin: the LixiLan-L randomized trial. Diabetes Care 2016:39:1972-80

19. Rosenstock J, Diamant M, Aroda VR, et al. Efficacy and safety of LixiLan, a titratable fixed-ratio combination of lixisenatide and insulin glargine, versus insulin glargine in type 2 diabetes inadequately controlled on metformin monotherapy: the LixiLan proof-of-concept randomized trial. Diabetes Care 2016;39:1579-86.
20. Rosenstock J, Aronson R, Grunberger G, et al. Benefits of LixiLan, a titratable fixed-ratio combination of insulin glargine plus lixisenatide, versus insulin glargine and lixisenatide monocomponents in type 2 diabetes inadequately controlled on oral agents: the LixiLan-O randomized trial. Diabetes Care 2016;39:2026-35.

21. IDF, 2017. IDF Diabetes Atlas, 8th Edition. Available: https://www. idf.org/component/attachments/attachments.html?id=1405\&task= download [Accessed 30 January 2019].

22. Woodward M, Patel A, Zoungas S, et al. Does glycemic control offer similar benefits among patients with diabetes in different regions of the world? Results from the ADVANCE trial. Diabetes Care $2011 ; 34: 2491-5$

23. ABDUL-GHANI M, PUCKETT CL, TRIPLITT CL, et al. Durable $\mathrm{HbA} 1 \mathrm{c}$ reduction with initial combination therapy with metformin/ pioglitazone/exenatide in subjects with new-onset diabetes-sixyear follow-up of the EDICT study. Diabetes 2018;67(Supplement 1).

24. Choi JH, Park CY, Cha BS, et al. Perception of clinicians and diabetic patients on the importance of postprandial glucose control and diabetes education status: a cross sectional survey. Diabetes Metab J 2012;36:120-7. 